\title{
The First Disease Episode: A Strategic Treatment Target to Prevent Cellulitis Relapses
}

\author{
Ioannis Chaniotakis Ioannis D. Bassukas \\ Department of Skin and Venereal Diseases, Faculty of Medicine, School of Health Sciences, University of \\ loannina, loannina, Greece
}

Cellulitis, a common bacterial skin infection, is characterized by a significant risk of readmission [1] and a high propensity to recur in situ [2]. Two hypotheses are discussed in the literature to explain this distinct behavior (Fig. 1). The traditional "re-infection hypothesis" interprets the increased recurrence risk as the consequence of local tissue modifications that are induced by a preceding disease episode and predispose to re-infection in the same skin area [2]. Recently, an alternative pathophysiologic explanation of recurring cellulitis has come into focus: recurrences may be local "relapses" of a latent infection, a consequence of pathogen survival and persistence in the already affected tissues [3]. The two major cellulitis pathogens Streptococcus pyogenes (GAS) and Staphylococcus aureus as well as many of the less frequent ones, like Escherichia coli, Pseudomonas aeruginosa, Acinetobacter baumannii, etc. [4], are competent to establish dormancy by at least two distinct mechanisms: intracellular survival and biofilm formation [5-7].

Currently, the standard of care for the uncomplicated cellulitis consists in relatively short courses (5 days) of beta-lactam antibiotics $[2,8]$. It is worth noting that in about $30 \%$ of GAS infections, penicillin treatment fails to eradicate the pathogen [9], a proportion quite similar to the fraction of patients suffering cellulitis recurrences

\footnotetext{
karger@karger.com

(C) 2020 S. Karger AG, Basel

www.karger.com/drm

Karger ${ }^{\prime \prime}=$
}

[10]. Nevertheless, to prevent infection latency, the antibacterial regimens need to (a) reach the pathogens in the biofilm and/or intracellular sanctuary and (b) target the reduced microorganism proliferation rate in the dormancy environment. Moreover, since after the first recurrence, the relapsing course of cellulitis seems to cannot be stopped anymore [10], we hypothesized that a main strategy to control relapses is to focus on the treatment of the first-ever disease episode. Favoring the hypothesis that disease recurrences correspond to relapses of a latent infection, we have modified our approach to hospitalized patients with cellulitis by introducing elongated therapeutic schemes (21 days) with antibiotics with known intracellular antibacterial activity (clindamycin or sequential daptomycin-roxithromycin). Details of treatment schemes, patients' core demographics, therapeutic outcomes, statistical methods, and authorities' permissions have been presented elsewhere $[11,12]$ and will not be repeated here. Herein, we integrated our observations of the "recurrence" rates of patients with first-ever lower extremity cellulitis episodes in order to highlight the impact of "eradication" schemes on the frequency of disease relapses. A total of 120 patients were initiated in one of three different 21-day antibiotic regimens: (1) clindamycin ( $n=83$ patients), (2) penicillin $(n=10)$, and (3) sequential 
Fig. 1. Cellulitis of the lower extremities: recurrence versus relapse. Already after the first cellulitis episode, tissue alterations may initiate a vicious circle of locally repeating disease episodes.

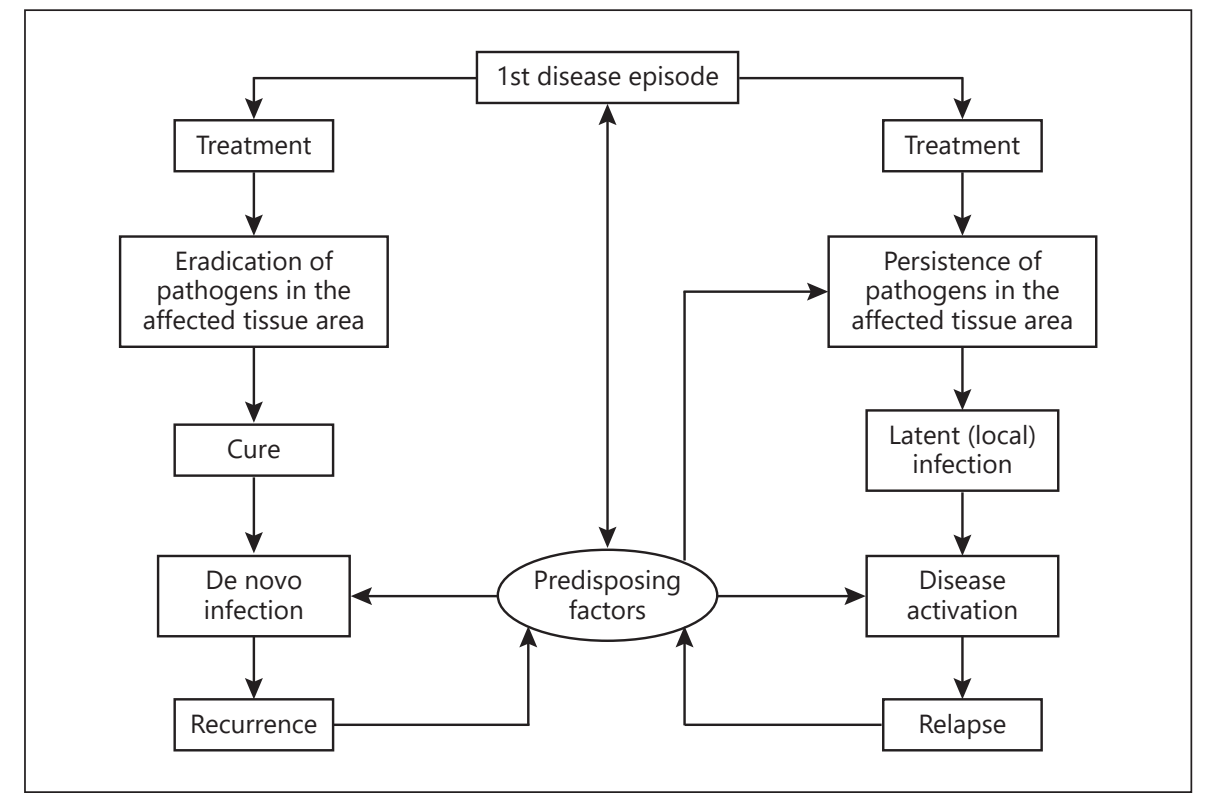

daptomycin (for the time of hospitalization) followed by oral administration of roxithromycin $(n=27)$. The cure rates were similarly high with all three regimens $(80 / 83=$ $96.4 \%$ in the clindamycin group, $9 / 10=90 \%$ in the penicillin group, and $26 / 27=96.3 \%$ in the daptomycin-based scheme; overall: $95.8 \%)$. More interesting, our data of the 115 patients (median age: 67 years; male: 60/115) who completed the above treatment schemes indicate low relapse rates only after treatment with antibiotics with intracellular activity. The 3-year recurrence rate was $6.6 \%$ (8.9\% at the 5-year follow-up) for the patients on clindamycin ( $n=80$ patients [11]) and $3.8 \%$ for those who completed the daptomycin-roxithromycin scheme $(n=$ 26 patients [12]). However, with $33.3 \%$, the 3 -year relapse rate in the smaller penicillin group $(n=9)$ was comparable to that known in the literature after conventional cellulitis treatment [10] and also significantly higher compared to the present clindamycin group $(p=0.012$ [11]). Lack of information on the causative agents at patients' level and of pathogens' susceptibility testing data are serious limitations in our studies.

In conclusion, the therapeutic efficacy of the "eradication" schemes seems to support the in situ pathogen dormancy as an important pathophysiologic mechanism of "relapsing" cellulitis. We would like to suggest that future clinical trials should focus on the treatment of the firstever disease episode as a promising intervention to prevent cellulitis relapses. Additionally, we would like to predict that microbiological findings supplemented by pathogen susceptibility test results, also for antibiotics ef- fective against intracellular microorganisms and pathogens in biofilms, is mandatory information for the rational approach to the treatment of relapsing cellulitis. Finally, since some cellulitis cases may be mixed-pathogen infections [4], future therapeutic trials should also highlight the impact of therapeutic approaches to modify the relapsing behavior of already recurring cellulitis.

\section{Key Message}

Targeting infection dormancy: the key intervention to prevent recurrence, the unmet need of cellulitis treatment?

\section{Disclosure Statement}

The authors have no conflicts of interest to declare.

\section{Funding Sources}

The authors did not receive any funding.

\section{Author Contributions}

I.C. analyzed data and wrote the first draft of the paper. I.D.B. designed the study and analyzed data. Both co-authors revised the manuscript for important intellectual content, approved the submitted version, and agree to be accountable for all aspects of the work. 


\section{References}

1 Garg A, Lavian J, Lin G, Sison C, Oppenheim M, Koo B. Clinical factors associated with readmission among patients with lower limb cellulitis. Dermatology. 2017;233(1):58-63.

2 Raff AB, Kroshinsky D. Cellulitis: A review. JAMA. 2016 Jul;316(3):325-37.

3 Jendoubi F, Rohde M, Prinz JC. Intracellular streptococcal uptake and persistence: A potential cause of erysipelas recurrence. Front Med (Lausanne). 2019 Jan;6:6.

4 Chaniotakis I, Gartzonika CG, Gaitanis G, Levidiotou-Stefanou S, Bassukas ID. Causality evaluation of bacterial species isolated from patients with community-acquired lower leg cellulitis. J Eur Acad Dermatol Venereol. 2016 Sep;30(9):1583-9.

5 Percival SL, Emanuel C, Cutting KF, Williams DW. Microbiology of the skin and the role of biofilms in infection. Int Wound J. 2012 Feb; 9(1):14-32.
6 Robino L, Scavone P, Araujo L, Algorta G, Zunino P, Pírez MC, et al. Intracellular bacteria in the pathogenesis of Escherichia coli urinary tract infection in children. Clin Infect Dis. 2014 Dec;59(11):e158-64.

7 Fiedler T, Köller T, Kreikemeyer B. Streptococcus pyogenes biofilms-formation, biology, and clinical relevance. Front Cell Infect Microbiol. 2015 Feb;5:15.

8 Brindle R, Williams OM, Barton E, Featherstone P. Assessment of antibiotic treatment of cellulitis and erysipelas: A systematic review and meta-analysis. JAMA Dermatol. 2019 Jun;155(9):1033. ; Epub ahead of print.

9 Ogawa T, Terao Y, Okuni H, Ninomiya K, Sakata H, Ikebe K, et al. Biofilm formation or internalization into epithelial cells enable Streptococcus pyogenes to evade antibiotic eradication in patients with pharyngitis. Microb Pathog. 2011 Jul-Aug;51(1-2):58-68.
10 Thomas KS, Crook AM, Nunn AJ, Foster KA, Mason JM, Chalmers JR, et al.; U.K. Dermatology Clinical Trials Network's PATCH I Trial Team. Penicillin to prevent recurrent leg cellulitis. N Engl J Med. 2013 May;368(18): 1695-703.

11 Chaniotakis I, Gaitanis G, Skandalis K, Alexis I, Bassukas ID. Reduced recurrence rate after a three week clindamycin for the initial lower limb cellulitis. J J Expt Derm Res. 2015 June; 1(3): 009. Available from: https://jacobspublishers.com/reduced-recurrence-rate-after-athree-week-clindamycin-for-the-initial-lower-limb-cellulitis/ [last accessed 2019 Apr 30].

12 Bassukas ID, Chaniotakis I, Gaitanis G. Daptomycin-based 3 week treatment scheme for the first cellulitis episode: low recurrence rate indicative of decreased recurrence propensity? Dermatol Ther (Heidelb). 2016 Mar;6(1): 77-80. 